PROCEEDINGS OF THE

AMERICAN MATHEMATICAL SOCIETY

Volume 138, Number 11, November 2010, Pages 3877-3889

S 0002-9939(2010)10428-8

Article electronically published on June 9, 2010

\title{
GENERALIZED YOUNG WALLS AND CRYSTAL BASES FOR QUANTUM AFFINE ALGEBRA OF TYPE $A$
}

\author{
JEONG-AH KIM AND DONG-UY SHIN
}

(Communicated by Gail R. Letzter)

\begin{abstract}
We give a new realization of the crystal $B(\infty)$ of $U_{q}^{-}\left(A_{n}^{(1)}\right)$ using generalized Young walls, a modified notion of Young walls of type $A_{n}^{(1)}$. Moreover, by the fact that the irreducible highest weight crystal $B(\lambda)$ lies in the crystal $B(\infty) \otimes R_{\lambda}$, we construct the crystal $B(\lambda)$ using generalized Young walls.
\end{abstract}

\section{INTRODUCTION}

In [7, Kashiwara developed the crystal basis theory for the negative part $U_{q}^{-}(\mathfrak{g})$ of the quantum groups $U_{q}(\mathfrak{g})$ and the integrable highest weight $U_{q}(\mathfrak{g})$-modules $V(\lambda)$ over $U_{q}(\mathfrak{g})$ with a dominant integral weight $\lambda$. Crystal basis theory is a nice combinatorial tool to understand the internal structure of integrable modules and the quantum group itself. For instance, one of the major goals in the representation theory is to find an explicit expression for the characters of representations, and this can be obtained by finding an explicit combinatorial description of crystal bases. Hence one of the basic and important problems in crystal basis theory is to realize the crystals explicitly using several combinatorial objects. In many articles, one can find several kinds of realizations of the crystal bases $B(\infty)$ of the negative part $U_{q}^{-}(\mathfrak{g})$ of $U_{q}(\mathfrak{g})$ and the crystal bases $B(\lambda)$ of the integrable highest weight $U_{q}(\mathfrak{g})$ modules $V(\lambda)$ using combinatorial objects (for example, [2, 3, 4, 5, 6, 8, 9, 10, 11]).

However, even though there are several combinatorial models realizing the crystal $B(\infty)$, there is no combinatorial model which is intuitive and easy to treat like the Young tableaux or Young walls that appeared in the realization of the highest weight crystal. Indeed, we need some effort to find the affine weights of the paths given in 3], and it is not easy to determine whether some sequence of nonnegative integers belongs to the polyhedral realization of $B(\infty)$ given in [11.

The main goal of this paper is to give new realizations of the crystals $B(\infty)$ and $B(\lambda)$ over the quantum affine algebra $U_{q}\left(A_{n}^{(1)}\right)$. More precisely, we introduce a new combinatorial object, generalized Young walls, which is a modified notion of the Young walls appearing in 2]. We give the rules and patterns for building generalized Young walls and the action of Kashiwara operators explicitly in terms of generalized

Received by the editors August 12, 2009 and, in revised form, January 20, 2010 and February 3, 2010

2010 Mathematics Subject Classification. Primary 17B37, 81R50.

This work was supported by the research fund of Hanyang University (HY-2009-O).

(C)2010 American Mathematical Society

Reverts to public domain 28 years from publication 
Young walls. Then we show that the set of reduced generalized Young walls is isomorphic to the crystal $B(\infty)$ of the negative part $U_{q}^{-}(\mathfrak{g})$ of the quantum affine Lie algebra of type $A$. As an application, the crystal $B(\infty)$ over the quantum finite algebra $U_{q}\left(A_{n}\right)$ in terms of generalized Young walls is obtained by characterizing the connected component containing the highest weight vector $u_{\infty}$ when we remove the 0 -arrows in the crystal graph $B(\infty)$ over $U_{q}\left(A_{n}^{(1)}\right)$. Finally, according to the fact that the highest weight crystal $B(\lambda)$ is isomorphic to the connected component containing $u_{\infty} \otimes r_{\lambda}$ in $B(\infty) \otimes R_{\lambda}$ [10, we give a realization of the crystal basis $B(\lambda)$ over $U_{q}\left(A_{n}^{(1)}\right)$ using generalized Young walls.

\section{Crystals}

Let $I$ be a finite index set. We set a Cartan datum $\left(A, P^{\vee}, P, \Pi^{\vee}, \Pi\right)$ as follows:

(i) generalized Cartan matrix $A=\left(a_{i j}\right)_{i, j \in I}$,

(ii) dual weight lattice $P^{\vee}=\left(\bigoplus_{i \in I} \mathbf{Z} h_{i}\right) \oplus\left(\bigoplus_{j=1}^{\operatorname{corank} A} \mathbf{Z} d_{j}\right)$,

(iii) weight lattice $P=\left\{\lambda \in \mathfrak{h}^{*} \mid \lambda\left(P^{\vee}\right) \subset \mathbf{Z}\right\}$, where $\mathfrak{h}=\mathbf{Q} \otimes_{\mathbf{Z}} P^{\vee}$,

(iv) the set of simple coroots $\Pi^{\vee}=\left\{h_{i} \mid i \in I\right\}$,

(v) the set of simple roots $\Pi=\left\{\alpha_{i} \mid i \in I\right\}$.

The simple roots are linearly independent and satisfy $\left\langle h_{i}, \alpha_{j}\right\rangle=a_{i j},\left\langle d_{s}, \alpha_{j}\right\rangle=0$ or 1 . The fundamental weights $\Lambda_{i}$ are defined by $\left\langle h_{j}, \Lambda_{i}\right\rangle=\delta_{i j},\left\langle d_{s}, \Lambda_{i}\right\rangle=0$. We also denote by $P^{+}=\left\{\lambda \in P \mid \lambda\left(h_{i}\right) \geq 0\right.$ for all $\left.i \in I\right\}$ the set of dominant integral weights.

Let us denote by $U_{q}(\mathfrak{g})$ the quantum group associated with the Cartan datum $\left(A, P^{\vee}, P, \Pi^{\vee}, \Pi\right)$, and let us denote by $U_{q}^{+}(\mathfrak{g})\left(\right.$ resp. $\left.U_{q}^{-}(\mathfrak{g})\right)$ the subalgebra of $U_{q}(\mathfrak{g})$ generated by the $e_{i}$ 's (resp. the $f_{i}$ 's). The crystal basis theory was developed for $U_{q}(\mathfrak{g})$-modules $M$ in the category $\mathcal{O}_{\text {int }}$ and the subalgebra $U_{q}^{-}(\mathfrak{g})$ of the quantum group $U_{q}(\mathfrak{g})$ by Kashiwara [7. Moreover, by abstracting the properties of crystal bases, Kashiwara et al. introduced the notion of abstract crystals [4.

Definition 1.1. An abstract crystal for $U_{q}(\mathfrak{g})$ or a $U_{q}(\mathfrak{g})$-crystal is a set $B$ together with the maps wt $: B \rightarrow P, \varepsilon_{i}, \varphi_{i}: B \rightarrow \mathbf{Z} \cup\{-\infty\}, \tilde{e}_{i}, \tilde{f}_{i}: B \rightarrow B \cup\{0\}(i \in I)$ such that for all $i \in I$ and $b \in B$,

(i) $\varphi_{i}(b)=\varepsilon_{i}(b)+\left\langle h_{i}, \operatorname{wt}(b)\right\rangle$,

(ii) $\operatorname{wt}\left(\tilde{e}_{i} b\right)=\operatorname{wt}(b)+\alpha_{i}$ if $\tilde{e}_{i} b \neq 0$,

(iii) $\operatorname{wt}\left(\tilde{f}_{i} b\right)=\operatorname{wt}(b)-\alpha_{i}$ if $\tilde{f}_{i} b \neq 0$,

(iv) $\varepsilon_{i}\left(\tilde{e}_{i} b\right)=\varepsilon_{i}(b)-1, \quad \varphi_{i}\left(\tilde{e}_{i} b\right)=\varphi_{i}(b)+1$ if $\tilde{e}_{i} b \neq 0$

(v) $\varepsilon_{i}\left(\tilde{f}_{i} b\right)=\varepsilon_{i}(b)+1, \quad \varphi_{i}\left(\tilde{f}_{i} b\right)=\varphi_{i}(b)-1$ if $\tilde{f}_{i} b \neq 0$,

(vi) $\tilde{f}_{i} b=b^{\prime}$ if and only if $\tilde{e}_{i} b^{\prime}=b$ for $b, b^{\prime} \in B$,

(vii) $\tilde{e}_{i} b=\tilde{f}_{i} b=0$ if $\varepsilon_{i}(b)=-\infty$.

Example 1.2. (1) For $\lambda \in P^{+}$, let $B(\lambda)$ be the crystal basis of the irreducible highest weight module $V(\lambda)$ over $U_{q}(\mathfrak{g})$. Then $B(\lambda)$ is a $U_{q}(\mathfrak{g})$-crystal, called the irreducible highest weight crystal.

(2) Let $U_{q}^{-}(\mathfrak{g})$ be the subalgebra of $U_{q}(\mathfrak{g})$ generated by the $\tilde{f}_{i}$ 's, and let $B(\infty)$ be the crystal graph of $U_{q}^{-}(\mathfrak{g})$. Then $B(\infty)$ is a $U_{q}(\mathfrak{g})$-crystal, and the highest weight vector is denoted by $u_{\infty}$.

(3) The singleton $R_{\lambda}=\left\{r_{\lambda}\right\}(\lambda \in P)$ is a $U_{q}(\mathfrak{g})$-crystal with

$$
\operatorname{wt}\left(r_{\lambda}\right)=\lambda, \quad \varepsilon_{i}\left(r_{\lambda}\right)=-\left\langle h_{i}, \lambda\right\rangle, \quad \varphi_{i}\left(r_{\lambda}\right)=0, \quad \tilde{e}_{i} r_{\lambda}=\tilde{f}_{i} r_{\lambda}=0 .
$$


Definition 1.3. A morphism $\psi: B_{1} \rightarrow B_{2}$ of crystals is a map $\psi: B_{1} \cup\{0\} \rightarrow$ $B_{2} \cup\{0\}$ satisfying the following conditions:

(i) $\psi(0)=0, \operatorname{wt}(\psi(b))=\mathrm{wt}(b)$,

(ii) $\varepsilon_{i}(\psi(b))=\varepsilon_{i}(b)$ and $\varphi_{i}(\psi(b))=\varphi_{i}(b)$ if $b \in B_{1}$ and $\psi(b) \in B_{2}$,

(iii) $\tilde{e}_{i} \psi(b)=\psi\left(b^{\prime}\right)$ if $b, b^{\prime} \in B_{1}, \psi(b), \psi\left(b^{\prime}\right) \in B_{2}$ and $\tilde{e}_{i} b=b^{\prime}$,

(iv) $\tilde{f}_{i} \psi(b)=\psi\left(b^{\prime}\right)$ if $b, b^{\prime} \in B_{1}, \psi(b), \psi\left(b^{\prime}\right) \in B_{2}$ and $\tilde{f}_{i} b=b^{\prime}$.

Let $B_{1}, B_{2}$ be crystals. Then the tensor product

$$
B_{1} \otimes B_{2}=\left\{b_{1} \otimes b_{2} ; b_{1} \in B_{1}, b_{2} \in B_{2}\right\}
$$

is also a crystal with the maps wt, $\varepsilon_{i}, \varphi_{i}, \tilde{e}_{i}, \tilde{f}_{i}$ given by

$$
\begin{aligned}
& \mathrm{wt}\left(b_{1} \otimes b_{2}\right)=\operatorname{wt}\left(b_{1}\right)+\operatorname{wt}\left(b_{2}\right), \\
& \varepsilon_{i}\left(b_{1} \otimes b_{2}\right)=\max \left\{\varepsilon_{i}\left(b_{1}\right), \varepsilon_{i}\left(b_{2}\right)-\left\langle h_{i}, \operatorname{wt}\left(b_{1}\right)\right\rangle\right\}, \\
& \varphi_{i}\left(b_{1} \otimes b_{2}\right)=\max \left\{\varphi_{i}\left(b_{2}\right), \varphi_{i}\left(b_{1}\right)+\left\langle h_{i}, \operatorname{wt}\left(b_{2}\right)\right\rangle\right\}, \\
& \tilde{e}_{i}\left(b_{1} \otimes b_{2}\right)= \begin{cases}\tilde{e}_{i} b_{1} \otimes b_{2} & \text { if } \varphi_{i}\left(b_{1}\right) \geq \varepsilon_{i}\left(b_{2}\right), \\
b_{1} \otimes \tilde{e}_{i} b_{2} & \text { if } \varphi_{i}\left(b_{1}\right)<\varepsilon_{i}\left(b_{2}\right),\end{cases} \\
& \tilde{f}_{i}\left(b_{1} \otimes b_{2}\right)= \begin{cases}\tilde{f}_{i} b_{1} \otimes b_{2} & \text { if } \varphi_{i}\left(b_{1}\right)>\varepsilon_{i}\left(b_{2}\right), \\
b_{1} \otimes \tilde{f}_{i} b_{2} & \text { if } \varphi_{i}\left(b_{1}\right) \leq \varepsilon_{i}\left(b_{2}\right) .\end{cases}
\end{aligned}
$$

We close this section by recalling the realization of the crystal basis $B(\infty)$ of the negative part $U_{q}^{-}\left(A_{n}^{(1)}\right)$ of $U_{q}\left(A_{n}^{(1)}\right)$ introduced in [3]. (Here, $U_{q}\left(A_{n}^{(1)}\right)$ is the affine quantum algebra of type $A$.)

Example 1.4. Let $B_{\infty}=\left\{b=\left(b_{0}, b_{1}, \ldots, b_{n}\right) \in \mathbf{Z}^{n+1} \mid \sum_{i=0}^{n} b_{i}=0\right\}$ and $b_{\infty}=$ $(0, \ldots, 0)$. Define

$$
\begin{aligned}
\operatorname{wt}(b) & =\left(b_{0}-b_{1}\right) \Lambda_{0}+\left(b_{1}-b_{2}\right) \Lambda_{1}+\cdots+\left(b_{n}-b_{0}\right) \Lambda_{n}, \\
\varepsilon_{i}(b) & =b_{i+1}(i \neq n), \quad \varepsilon_{n}(b)=b_{0}, \quad \varphi_{i}(b)=b_{i}, \\
\tilde{f}_{i} b & =\left\{\begin{array}{l}
\left(b_{0}, \ldots, b_{i}-1, b_{i+1}+1, \ldots, b_{n}\right), \quad i \neq n, \\
\left(b_{0}+1, b_{1}, \ldots, b_{n}-1\right), \quad i=n,
\end{array}\right. \\
\tilde{e}_{i} b & =\left\{\begin{array}{l}
\left(b_{0}, \ldots, b_{i}+1, b_{i+1}-1, \ldots, b_{n}\right), \quad i \neq n, \\
\left(b_{0}-1, b_{1}, \ldots, b_{n}+1\right), \quad i=n .
\end{array}\right.
\end{aligned}
$$

A path in $B_{\infty}$ is a sequence $\mathbf{p}=\left(p_{k}\right)_{k=1}^{\infty}=\cdots \otimes p_{k} \otimes \cdots \otimes p_{1}$ such that $p_{k} \in B_{\infty}$ for all $k \geq 1$ and $p_{k}=0$ for $k \gg 0$. We denote by $\mathcal{P}(\infty)$ the set of all paths in $B_{\infty}$. Then there is a $U_{q}\left(A_{n}^{(1)}\right)$-crystal isomorphism

$$
\psi: B(\infty) \longrightarrow \mathcal{P}(\infty)
$$

given by $u_{\infty} \longmapsto \psi\left(u_{\infty}\right)=\cdots \otimes b_{\infty} \otimes b_{\infty} \otimes b_{\infty}[3]$.

\section{Generalized Young walls}

In this section, we introduce the notion of generalized Young walls, which will be used to realize the crystal $B(\infty)$ for the quantum affine Lie algebra $U_{q}\left(A_{n}^{(1)}\right)$. In 2], Kang introduced the notion of Young walls and constructed the crystal bases $B(\lambda)$ of the basic representation $V(\lambda)$ over classical quantum affine Lie algebras $U_{q}(\mathfrak{g})$. The notion of generalized Young walls is a generalization of the notion of a Young wall of type $A_{n}^{(1)}$. 
Let $\mathcal{B}$ be a board with coloring as follows:

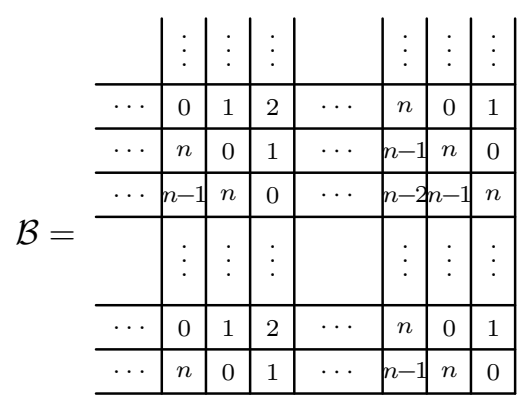

Here, the color of the $j$-th site from the bottom of the $i$-th column from the right of $\mathcal{B}$ is $j-i(\bmod n+1)$.

Definition 2.1. The generalized Young walls are constructed of $i$-colored boxes $i(i \in I)$ on the board $\mathcal{B}$ under the following building rules:

(i) The colored boxes should be located according to the colors of the sites of the board $\mathcal{B}$.

(ii) The colored boxes should be put in rows. That is, in each row, one puts colored boxes from right to left.

Example 2.2. Let

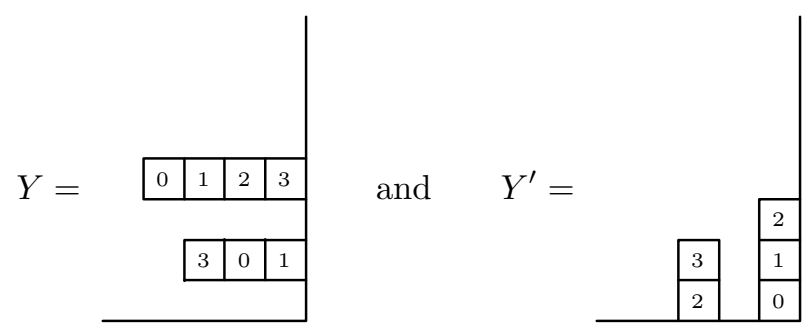

Then clearly $Y$ is a generalized Young wall for $U_{q}\left(A_{3}^{(1)}\right)$, but $Y^{\prime}$ is not.

Remark 2.3. Indeed, the Young walls are 3-dimensional objects. But, Young walls realizing the crystal bases of basic representations can be regarded as 2-dimensional objects. The generalized Young walls are refined versions of the Young walls appearing in the crystal bases of basic representations of quantum affine algebras of type $A_{n}^{(1)}$. (Also, they are generalizations of the colored Young diagrams introduced in [1.)

Definition 2.4. A generalized Young wall is said to be proper if for any $p>q$ and $p-q \equiv 0(\bmod n+1)$, the number of boxes in the $p$-th row from the bottom is equal to or smaller than that in the $q$-th row from below. 
Example 2.5. Let

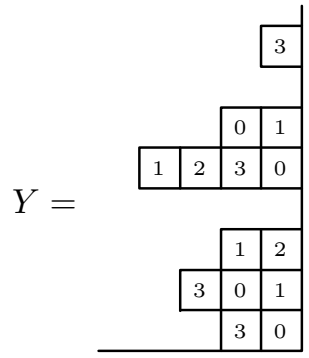

be a generalized Young wall for $U_{q}\left(A_{3}^{(1)}\right)$. Then we can easily find that the number of boxes in the fifth row is greater than the number of boxes in the first row. Thus, it is not proper.

Definition 2.6. (a) The leftmost box in each row of a proper generalized Young wall is called a removable box. Also, if it is $i$-colored, then it is called a removable $i$-box.

(b) The left site of the leftmost box in each row of a proper generalized Young wall is called an admissible slot. If there is no box for some row, the rightmost site of that row is an admissible slot. Also, if it is $i$-colored, then it is called an $i$-admissible slot.

Let $\mathcal{F}(\infty)$ be the set of all proper generalized Young walls. We define the action of Kashiwara operators $\tilde{e}_{i}, \tilde{f}_{i}(i \in I)$ on $\mathcal{F}(\infty)$. Fix $i \in I$ and let $Y \in \mathcal{F}(\infty)$ be a proper generalized Young wall. Let $y_{1}, y_{2}, y_{3}, \ldots$ be the removable $i$-boxes or $i$-admissible slots from left to right and from bottom to top. At first, to each $y_{j}$ $(j \in \mathbf{N})$, we assign its $i$-signature $\operatorname{sgn}_{i}\left(y_{j}\right)$ as $-($ resp. +$)$ if it is a removable box (resp. admissible slot). From the sequence $\left(\operatorname{sgn}_{i}\left(y_{1}\right), s g n_{i}\left(y_{2}\right), \ldots\right)$ of +'s and -'s, cancel out every $(+,-)$-pair to obtain a sequence of -'s followed by + 's, reading from left to right. This sequence is called the $i$-signature of $Y$. (Note that there is an infinite number of + 's in the $i$-signature of $Y$ because infinitely many $i$-admissible slots exist in the first column.)

We define $\tilde{f}_{i} Y$ to be the proper generalized Young wall obtained from $Y$ by stacking the $i$-colored box to the $i$-admissible slot corresponding to the leftmost + in the $i$-signature of $Y$. We define $\tilde{e}_{i} Y$ to be the proper generalized Young wall obtained from $Y$ by eliminating the removable $i$-box corresponding to the rightmost - in the $i$-signature of $Y$. If there is no - in the $i$-signature of $Y$, we define $\tilde{e}_{i} Y=0$. We also define the maps

$$
\text { wt }: \mathcal{F}(\infty) \rightarrow P, \varepsilon_{i}: \mathcal{F}(\infty) \rightarrow \mathbf{Z}, \varphi_{i}: \mathcal{F}(\infty) \rightarrow \mathbf{Z}
$$

by

$$
\begin{aligned}
\operatorname{wt}(Y) & =-\sum_{i \in I} k_{i} \alpha_{i}, \\
\varepsilon_{i}(Y) & =\text { the number of -'s in the } i \text {-signature of } Y, \\
\varphi_{i}(Y) & =\varepsilon_{i}(Y)+\left\langle h_{i}, \operatorname{wt}(Y)\right\rangle,
\end{aligned}
$$

where $k_{i}$ is the number of $i$-colored boxes in $Y$. Then it is easy to see that $\left(\mathcal{F}(\infty)\right.$, wt $\left., \varepsilon_{i}, \varphi_{i}, \tilde{e}_{i}, \tilde{f}_{i}\right)$ is an affine crystal. 
Example 2.7. Let

$$
Y=\begin{array}{|l|l|} 
& 3 \\
\hline 1 & 2 \\
\hline 0 & 1 \\
\hline
\end{array}
$$

be a proper generalized Young wall for $U_{q}\left(A_{3}^{(1)}\right)$. Then it is easy to see that the third site from the bottom of the third column from the right and the $(4 k+1)$-st $(k \geq 0)$ sites from the bottom of the rightmost column are 0 -admissible slots, and the 0 -box in the second column from the right is a removable 0-box. The following describes the 0 -admissible slots and removable 0-boxes of $Y$ :

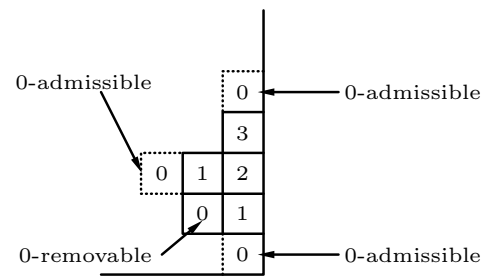

Thus, the 0 -signature of $Y$ is $(\underbrace{+}_{3^{\text {rd }}}, \underbrace{-}_{2^{\text {nd }}}, \underbrace{+,+,+, \cdots}_{1^{\text {st }}})$, and so we have

$$
\tilde{f}_{0} Y=\begin{array}{|l|l|}
\cline { 2 - 2 } & 3 \\
\hline 1 & 2 \\
\hline 0 & 1 \\
\hline & 0 \\
\hline
\end{array}
$$

Here, the under-braced $1^{\text {st }}, 2^{\text {nd }}$ and $3^{\text {rd }}$ in the 0 -signature mean that the entries in the 0-signature correspond to the admissible slots and removable blocks on the first, second and third columns of $Y$, respectively.

Similarly, we have

$$
\tilde{f}_{1} Y=\begin{array}{|l|l|}
\hline & 3 \\
\hline 1 & 2 \\
\hline 0 & 1 \\
\hline
\end{array}, \quad \tilde{f}_{2} Y=\begin{array}{|l|l|}
\hline 2 & 3 \\
\hline 1 & 2 \\
\hline 0 & 1 \\
\hline
\end{array} \text { and } \tilde{f}_{3} Y=\begin{array}{|r|r|}
\hline 1 \\
\hline
\end{array}
$$

Definition 2.8. (a) The $k$-th column $Y_{k}$ of a generalized Young wall $Y$ from the right is said to contain a removable $\delta$ if we may remove one $i$-colored box for all $i \in I$ from $Y_{k}$ and still obtain a generalized Young wall. That is, $Y_{k}$ contains a removable $\delta$ if

$$
a_{i-1}(k+1)<a_{i}(k) \text { for all } i \in \mathbf{Z}_{n+1}=\mathbf{Z} /(n+1) \mathbf{Z} .
$$

Here, $a_{i}(k)\left(i \in \mathbf{Z}_{n+1}, k \geq 1\right)$ is the number of $i$-colored boxes in the $k$-th column $Y_{k}$.

(b) A generalized Young wall is said to be reduced if it has no column containing a removable $\delta$. 
Example 2.9. Let

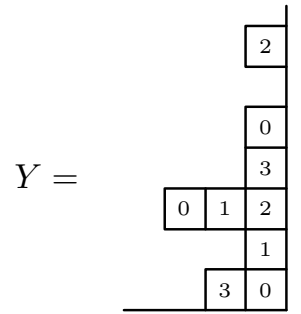

be a generalized Young wall for $U_{q}\left(A_{3}^{(1)}\right)$. Then it is easy to see that the first column $Y_{1}$ has a removable $\delta$. Moreover, the generalized Young wall $Y \backslash \delta$ obtained from $Y$ by deleting the removable $\delta$ in $Y_{1}$ is

$$
Y \backslash \delta=\begin{array}{|l|l|l|}
\hline 0 & 1 & 2 \\
\hline & & \\
\hline & 3 & 0 \\
\hline
\end{array}
$$

and it is reduced.

Let $\mathcal{Y}(\infty)$ be the set of all reduced proper generalized Young walls.

Proposition 2.10. For any $Y \in \mathcal{Y}(\infty)$, we have

$$
\tilde{f}_{i} Y \in \mathcal{Y}(\infty) \quad \text { and } \quad \tilde{e}_{i} Y \in \mathcal{Y}(\infty) \cup\{0\} .
$$

Proof. Let $Y \in \mathcal{Y}(\infty)$ and $i \in I$. By definition of the Kashiwara operator acting on the set $\mathcal{Y}(\infty), \tilde{f}_{i} Y \neq 0$. Suppose that $\tilde{f}_{i} Y$ is obtained from $Y$ by stacking an $i$-box in the $k$-th column $Y_{k}$ of $Y$. If $\tilde{f}_{i} Y \notin \mathcal{Y}(\infty)$, then it means that $Y$ is reduced but $\tilde{f}_{i} Y$ is not reduced. Note that since the $k$-th column of $\tilde{f}_{i} Y$ has a removable $\delta$, there must be an $i$-admissible slot in the $(k+1)$-st column of $Y$. But, since $\tilde{f}_{i} Y$ is obtained by adding an $i$-box in the $k$-th column of $Y$, there must be a removable $i$-box in the $k$-th column of $Y$. Therefore, $Y$ also has a removable $\delta$ in the $k$-th column. This is a contradiction.

Suppose that $\tilde{e}_{i} Y \neq 0$, and it is obtained from $Y$ by removing an $i$-colored box in the $k$-th column of $Y$. If $\tilde{e}_{i} Y \notin \mathcal{Y}(\infty)$, that is, $Y_{k-1}$ in $\tilde{e}_{i} Y$ has a removable $\delta$, then there is no $i$-admissible slot in the $k$-th column and there is a removable $i$-box in the $(k-1)$-st column of $Y$. It is a contradiction by definition of the Kashiwara operator $\tilde{e}_{i}$.

\section{Realization of the crystal $B(\infty)$ over $U_{q}\left(A_{n}^{(1)}\right)$}

In this section, we will give a new realization of the crystal $B(\infty)$ over $U_{q}\left(A_{n}^{(1)}\right)$ in terms of reduced proper generalized Young walls.

Let $\mathcal{P}(\infty)$ be the set of all paths in $B_{\infty}$ given in Example 1.4, and let $\mathcal{Y}(\infty)$ be the set of all reduced proper generalized Young walls. Then we have

Theorem 3.1. There is a $U_{q}\left(A_{n}^{(1)}\right)$-crystal isomorphism

$$
\Phi: \mathcal{Y}(\infty) \stackrel{\sim}{\longrightarrow} \mathcal{P}(\infty) \cong B(\infty)
$$

sending $Y_{\infty}$ to $\cdots \otimes b_{\infty} \otimes \cdots \otimes b_{\infty}$. 
Proof. Let $Y$ be a reduced proper generalized Young wall in $\mathcal{Y}(\infty)$. We define $\Phi(Y)=\left(\Phi(Y)(k) ; k \in \mathbf{Z}_{>0}\right)$ to be the path such that

$$
\Phi(Y)(k)=\left(a_{n}(k)-a_{0}(k), a_{0}(k)-a_{1}(k), \ldots, a_{n-1}(k)-a_{n}(k)\right),
$$

where $a_{i}(k)$ is the number of $i$-boxes in the $k$-th column $Y_{k}$ in $Y$. Then it is clear that $\Phi(Y)$ belongs to $\mathcal{P}(\infty)$.

Conversely, let $b$ be a path in $\mathcal{P}(\infty)$. Then it is expressed as

$$
b=\left(b(k)=\left(b_{0}(k), \ldots, b_{n}(k)\right) ; \sum_{i \in I} b_{i}(k)=0 \text { for each } k \in \mathbf{Z}_{>0}\right) .
$$

For each $k>0$ and $t_{k} \in \mathbf{Z}$, if we set

$$
\begin{aligned}
a_{0}(k) & =t_{k}+b_{n}(k)+b_{n-1}(k)+\cdots+b_{1}(k), \\
a_{1}(k) & =t_{k}+b_{n}(k)+b_{n-1}(k)+\cdots+b_{2}(k), \\
& \vdots \\
a_{n-1}(k) & =t_{k}+b_{n}(k), \\
a_{n}(k) & =t_{k},
\end{aligned}
$$

then $a_{i}(k) \geq 0$ for sufficiently large $t_{k}$. Moreover, it is clear that there exists a unique sequence $\left(t_{k} ; k \in \mathbf{Z}_{>0}\right)$ of integers such that the family of sequences $\left\{\left(a_{0}(k), \ldots, a_{n}(k)\right) ; k \in \mathbf{Z}_{>0}\right\}$ satisfies

(i) $a_{i}(k)=0$ for all $i \in I$ and $k \gg 0$,

(ii) $a_{i-1}(k+1) \leq a_{i}(k)$ for $i \in I$ and $k \in \mathbf{Z}_{>0}$,

(iii) for each $k \in \mathbf{Z}_{>0}$, there exist $i \in I$ such that $a_{i-1}(k+1)=a_{i}(k)$.

Now, we define $\Psi(b)$ to be the reduced proper generalized Young wall such that the number of $i$-colored boxes in the $k$-th column in $\Psi(b)$ is $a_{i}(k)$ given by the above family of sequences. Then it is clear that $\Phi$ and $\Psi$ are inverses of each other.

Note that the number of $i$-admissible slots (resp. removable $i$-boxes) in the $k$-th (resp. $(k-1)$-st) column is $a_{i+1}(k-1)-a_{i}(k)$ (resp. $\left.a_{i}(k-1)-a_{i-1}(k)\right)$. Therefore, by definition of the Kashiwara operators on the sets of proper generalized Young walls and paths, respectively, it is easy to see that $\Phi$ is a crystal morphism.

Remark 3.2. Let $Y$ be a reduced proper generalized Young wall and let $a_{i}(k)$ be the number of $i$-colored boxes in the $k$-th $(k>0)$ column $Y_{k}$ of $Y$. Then by Example 1.4 and Theorem 3.1, $\varphi(Y)$ can be rewritten as

$$
\varphi(Y)=\max \left\{\sum_{1 \leq k \leq n}\left(a_{i-1}(k)-a_{i}(k)\right) \mid n>0\right\} .
$$

This definition of $\varphi(Y)$ for $Y \in \mathcal{Y}(\infty)$ will be used to prove Theorem 4.1 .

Example 3.3. The following is the top part of $\mathcal{Y}(\infty)$ over $U_{q}\left(A_{2}^{(1)}\right)$. 


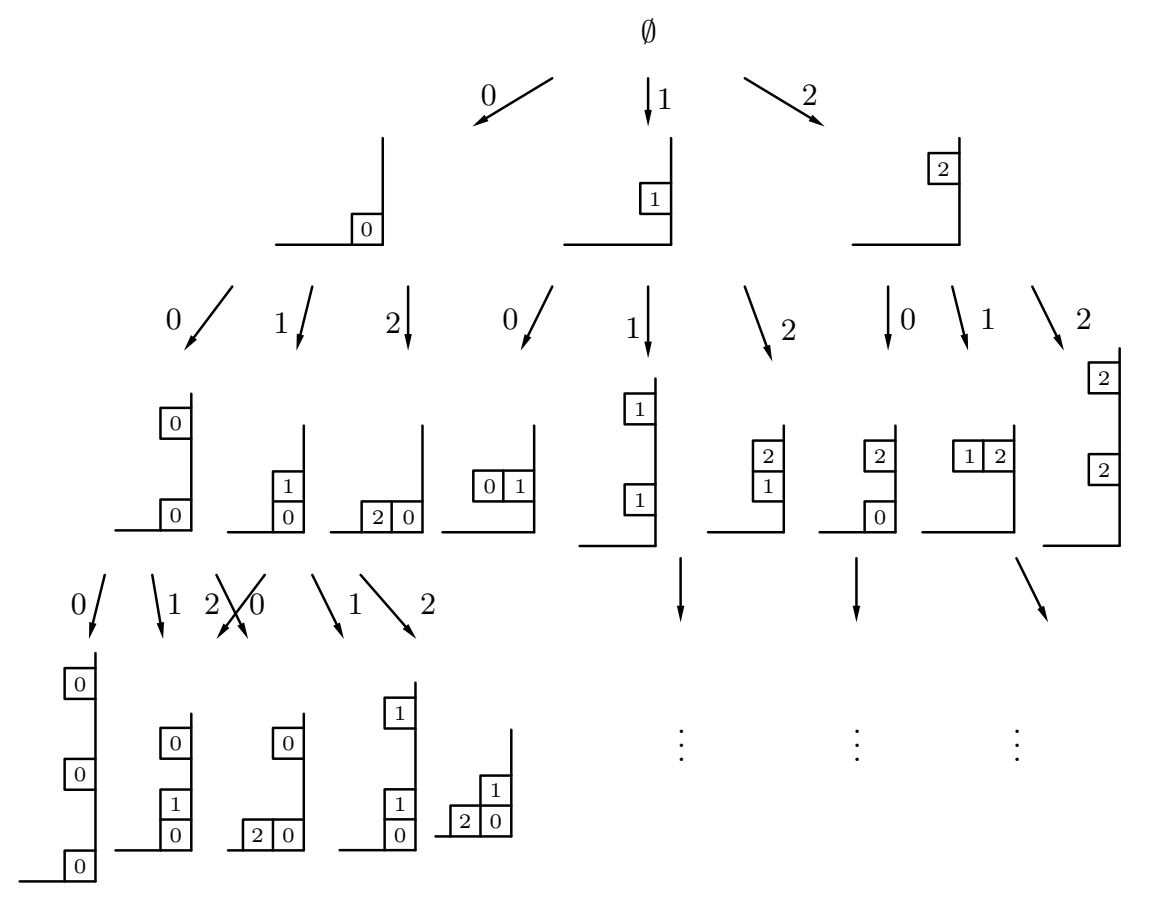

Corollary 3.4. Let $\mathfrak{g}=A_{n}^{(1)}$. Then we have

$$
\mathcal{F}(\infty) \cong \bigcup_{m \geq 0}\left(B(\infty) \otimes T_{-m \delta}\right)^{\oplus p(m)}
$$

where $p(m)$ is the partition function, and $B(\infty) \otimes T_{-m \delta}$ is the crystal basis of the Verma module $M(-m \delta)$ with highest weight $-m \delta$.

Proof. First, by definition of the Kashiwara operator $\tilde{e}_{i}$, it is easy to see that the following is the set of all maximal vectors in $\mathcal{F}(\infty)$ :

$$
\left\{Y \in \mathcal{F}(\infty) \mid a_{0}(k)=\cdots=a_{n}(k) \text { for all } k\right\} .
$$

For each maximal vector $Y$, if we set $a_{i}(k)-a_{i}(k+1)=b_{i}(k)$ for all $k \geq 1$, we associate a partition as follows:

$$
\left(1^{b_{i}(1)}, \ldots, k^{b_{i}(k)}, \ldots\right) \vdash \sum_{k \geq 1} k b_{i}(k) .
$$

Note that

$$
\operatorname{wt}(Y)=-\sum_{k \geq 1} k b_{i}(k) \delta
$$

Therefore, we have the results.

We know that the crystal $B(\infty)$ over $U_{q}\left(A_{n}\right)$ lies in the crystal graph $B(\infty)$ over $U_{q}\left(A_{n}^{(1)}\right)$ as the connected component containing $u_{\infty}$ if we remove the 0 -arrows in the crystal graph $B(\infty)$ over $U_{q}\left(A_{n}^{(1)}\right)$. Let $Y$ be a reduced proper generalized 
Young wall in the connected component $\mathcal{Y}^{\text {fin }}(\infty)$ containing $\emptyset$. Since $a_{0}(k)=0$ for all $k \in \mathbf{Z}_{>0}$, we have

$$
a_{i}(k)=0 \quad \text { unless } i=1, \ldots, n \text { and } k=1, \ldots, n-i+1 .
$$

Therefore, we have

Corollary 3.5. The crystal graph $\mathcal{Y}^{\text {fin }}(\infty)$ containing $Y_{\infty}$ over $U_{q}\left(A_{n}\right)$ is realized as the set of reduced proper generalized Young walls $Y$ such that

$$
a_{i}(k)=0 \text { unless } i=1, \ldots, n \text { and } k=1, \ldots, n-i+1 .
$$

That is, $\mathcal{Y}^{\text {fin }}(\infty)$ can be regarded as a subset of the set of $(n \times n)$ upper triangular matrices $\left(b_{i j}\right)_{n \times n}$ such that for each $i=1, \ldots, n$,

$$
b_{i j} \geq b_{i, j+1} \geq \cdots \geq b_{i, n-i+1} \text { and } b_{i, n-i+2}=b_{i, n-i+3}=\cdots=b_{i n}=0 .
$$

\section{IRREDUCIBLE HIGHEST WEIGHT CRYSTALS}

Let $B(\infty)$ be the crystal basis of the negative part $U_{q}^{-}(\mathfrak{g})$ of $U_{q}(\mathfrak{g})$, and let $R_{\lambda}=\left\{r_{\lambda}\right\}(\lambda \in P)$ be the singleton crystal with

$$
\operatorname{wt}\left(r_{\lambda}\right)=\lambda, \quad \varepsilon_{i}\left(r_{\lambda}\right)=-\left\langle h_{i}, \lambda\right\rangle, \quad \varphi_{i}\left(r_{\lambda}\right)=0, \quad \tilde{e}_{i} r_{\lambda}=\tilde{f}_{i} r_{\lambda}=0 .
$$

The highest weight crystal $B(\lambda)$ associated with the irreducible highest weight module $V(\lambda)\left(\lambda \in P^{+}\right)$over $U_{q}(\mathfrak{g})$ lies in the crystal $B(\infty) \otimes R_{\lambda}$ as the connected component containing $u_{\infty} \otimes r_{\lambda}$ [10. Therefore, it is natural to seek the explicit description of the elements belonging to the connected component $C\left(\emptyset \otimes r_{\lambda}\right)(\cong$ $B(\lambda)$ ) containing $\emptyset \otimes r_{\lambda}$ in $\mathcal{Y}(\infty) \otimes R_{\lambda}\left(\cong B(\infty) \otimes R_{\lambda}\right)$.

Let $\lambda=\Lambda_{i_{1}}+\cdots+\Lambda_{i_{l}}\left(0 \leq i_{1} \leq \cdots \leq i_{l} \leq n\right)$ be a dominant integral weight of level $l>0$. Let $Y$ be a reduced proper generalized Young wall in $\mathcal{Y}(\infty)$ and let $a_{i}(k)$ be the number of $i$-colored boxes in the $k$-th column $Y_{k}$ in $Y$. Then we have:

Theorem 4.1. The connected component $C\left(\emptyset \otimes r_{\lambda}\right)$ isomorphic to the highest weight $U_{q}\left(A_{n}^{(1)}\right)$-crystal $B(\lambda)$ is the set consisting of $Y \otimes r_{\lambda} \in \mathcal{Y}(\infty) \otimes R_{\lambda}$ satisfying the following condition:

for each $k \in \mathbf{Z}_{>0}$, if there exists $j \in I$ such that $a_{j}(k)-a_{j-1}(k)>0$, then for some $p=1, \ldots, l$,

$$
j+k \equiv i_{p}+1(\bmod n+1) \text { and } a_{j}(k)-a_{j-1}(k) \leq \lambda\left(h_{i_{p}}\right) .
$$

Proof. Let $Y \otimes r_{\lambda}$ be an element of $\mathcal{Y}(\infty) \otimes R_{\lambda}$ satisfying (4.1). Suppose that $\tilde{f}_{i}\left(Y \otimes r_{\lambda}\right)=\tilde{f}_{i} Y \otimes r_{\lambda} \neq 0$. Then by Remark 3.2.

$$
\varphi_{i}(Y)=\max \left\{\sum_{1 \leq k \leq n}\left(a_{i-1}(k)-a_{i}(k)\right) \mid n>0\right\}>-\lambda\left(h_{i}\right) .
$$

We assume that $\tilde{f}_{i} Y$ is obtained from $Y$ by stacking an $i$-colored box to the $k_{0}$-th column of $Y$ for some $k_{0}>0$. Then by definition of the Kashiwara operator $\tilde{f}_{i}$,

$$
a_{i+1}\left(k_{0}-1\right)-a_{i}\left(k_{0}\right)>a_{i}\left(k_{0}-1\right)-a_{i-1}\left(k_{0}\right) .
$$


First, suppose that $a_{i}\left(k_{0}\right)-a_{i-1}\left(k_{0}\right)=0$ in $Y$. Then $a_{i}\left(k_{0}\right)-a_{i-1}\left(k_{0}\right)=1>0$ in $\tilde{f}_{i} Y$, and by (4.2),

$$
a_{i+1}\left(k_{0}-1\right)>a_{i}\left(k_{0}-1\right) \quad \text { in } Y \text { and } \tilde{f}_{i} Y
$$

Therefore, by (4.1), there exists $p$ such that

$$
(i+1)+\left(k_{0}-1\right) \equiv i_{p}+1(\bmod n+1)
$$

which implies that

$$
i+k_{0} \equiv i_{p}+1(\bmod n+1)
$$

Second, suppose that $a_{i}\left(k_{0}\right)-a_{i-1}\left(k_{0}\right)=\lambda\left(h_{i_{p}}\right)>0$ for some $p=1, \ldots, l$ in $Y$. Then by (4.2),

$$
a_{i+1}\left(k_{0}-1\right)-a_{i}\left(k_{0}-1\right)>a_{i}\left(k_{0}\right)-a_{i-1}\left(k_{0}\right)=\lambda\left(h_{i_{p}}\right) .
$$

But, since $(i+1)+\left(k_{0}-1\right) \equiv i_{p}+1(\bmod n+1)$, it contradicts (4.1). Therefore, $\tilde{f}_{i}\left(Y \otimes r_{\lambda}\right)$ satisfies (4.1).

Similarly, it is easy to see that $\tilde{e}_{i}\left(Y \otimes r_{\lambda}\right) \neq 0(i \in I)$ satisfies (4.1).

Now, we will show that for any $Y \otimes r_{\lambda} \in \mathcal{Y} \otimes R_{\lambda}$ satisfying (4.1), if $\tilde{e}_{i}\left(Y \otimes r_{\lambda}\right)=0$ for all $i \in I$, then $Y=\emptyset$. It suffices to show that

$$
\tilde{e}_{i}\left(Y \otimes r_{\lambda}\right)=\tilde{e}_{i} Y \otimes r_{\lambda} \text { for all } Y \in \mathcal{Y}_{\infty}(\lambda)
$$

First, if $a_{i-1}(1)-a_{i}(1) \geq 0$, then

$$
\begin{aligned}
\varphi_{i}(Y) & =\max \left\{\sum_{1 \leq k \leq n}\left(a_{i-1}(k)-a_{i}(k)\right) \mid n>0\right\} \\
& \geq a_{i-1}(1)-a_{i}(1) \geq 0 \geq-\lambda\left(h_{i}\right),
\end{aligned}
$$

which implies (4.3). Second, consider the case that $a_{i-1}(1)-a_{i}(1)<0$. Then there exists $p(p=1, \ldots, l)$ such that $i=i_{p}$ and

$$
a_{i}(1)-a_{i-1}(1) \leq \lambda\left(h_{i}\right)
$$

Therefore,

$$
\begin{aligned}
\varphi_{i}(Y) & =\max \left\{\sum_{1 \leq k \leq n}\left(a_{i-1}(k)-a_{i}(k)\right) \mid n>0\right\} \\
& \geq a_{i-1}(1)-a_{i}(1) \geq-\lambda\left(h_{i}\right)
\end{aligned}
$$

which completes the proof.

Example 4.2. The following is the top part of $\mathcal{Y}_{\infty}\left(\Lambda_{1}\right)$ over $U_{q}\left(A_{2}^{(1)}\right)$. 


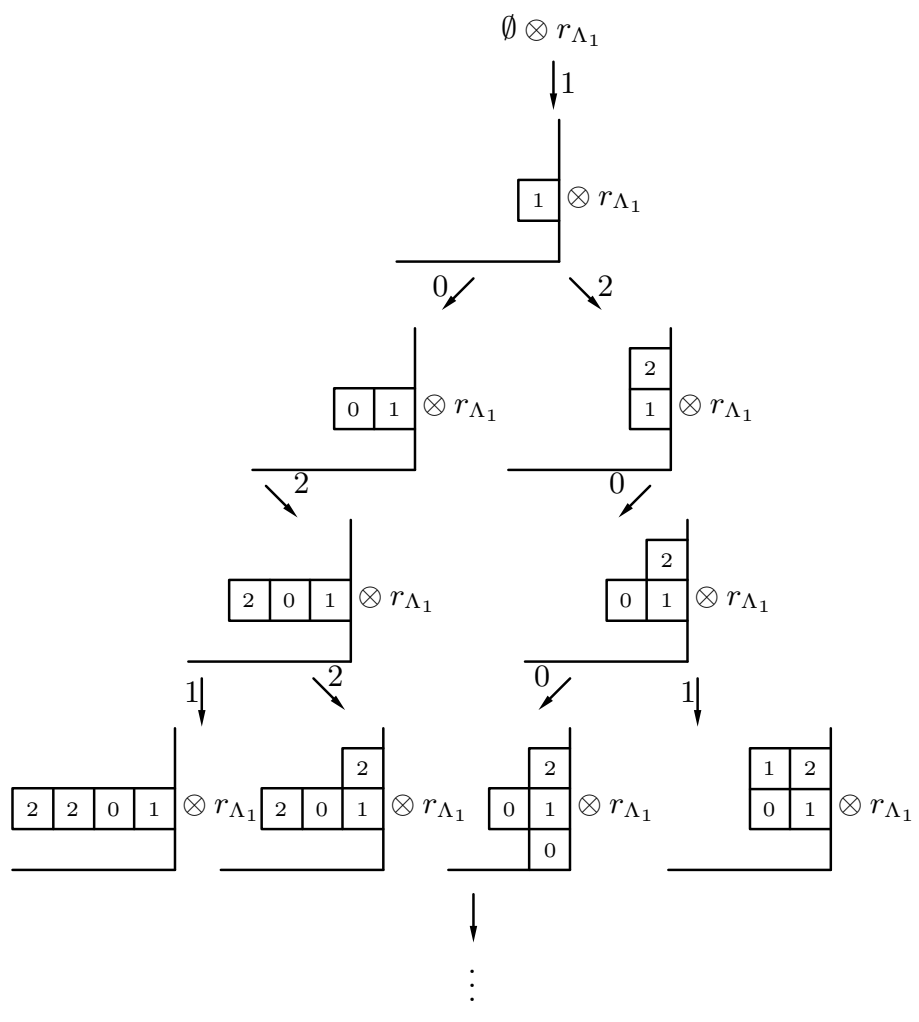

\section{ACKNOWLEDGMENT}

The authors would like to express their sincere gratitude to Professor S.-J. Kang for his interest in this work and for his encouragement.

\section{REFERENCES}

[1] M. Jimbo, K. C. Misra, T. Miwa, M. Okado, Combinatorics of representations of $U_{q}(\widehat{s l}(n))$ at $q=0$, Comm. Math. Phys. 136 (1991), 543-566. MR.1099695 (93a:17015)

[2] S.-J. Kang, Crystal bases for quantum affine algebras and combinatorics of Young walls, Proc. London Math. Soc. (3) 86 (2003), 29-69. MR.1971463(2004c:17028)

[3] S.-J. Kang, M. Kashiwara, K. C. Misra, Crystal bases of Verma modules for quantum affine Lie algebras, Compositio Math. 92 (1994), 299-325. MR1286129(95h:17016)

[4] S.-J. Kang, M. Kashiwara, K. C. Misra, T. Miwa, T. Nakashima, A. Nakayashiki, Affine crystals and vertex models, Int. J. Mod. Phys. A. Suppl. 1A (1992), 449-484. MR.1187560 (94a:17008)

[5] S.-J. Kang, J.-A. Kim, D.-U. Shin, Monomial realization of crystal bases for special linear Lie algebras, J. Algebra 274 (2004), 629-642. MR2043368 (2005a:17010)

[6] S.-J. Kang, J.-A. Kim, D.-U. Shin, Modified Nakajima monomials and the crystal $B(\infty)$, J. Algebra 308 (2007), 524-535. MR2295073 (2008b:17020)

[7] M. Kashiwara, On crystal bases of the Q-analogue of universal enveloping algebras, Duke Math. J. 63 (1991), 465-516. MR.1115118 (93b:17045)

[8] M. Kashiwara, T. Nakashima, Crystal graphs for representations of the q-analogue of classical Lie algebras, J. Algebra 165 (1994), 295-345. MR.1273277 (95c:17025)

[9] P. Littelmann, Paths and root operators in representation theory, Ann. of Math. (2) 142 (1995), 499-525. MR:1356780 (96m:17011) 
[10] T. Nakashima, Polyhedral realizations of crystal bases for integrable highest weight modules, J. Algebra 219 (1999), 571-597. MR1706829 (2000g:17020)

[11] T. Nakashima, A. Zelevinsky, Polyhedral realizations of crystal bases for quantized KacMoody algebras, Adv. Math. 131 (1997), 253-278. MR1475048 (98m:17023)

Department of Mathematics, University of Seoul, Seoul, 130-743, Korea

E-mail address: jakim@uos.ac.kr

Department of Mathematics Education, Hanyang University, Seoul 133-791, Korea

E-mail address: dushin@hanyang.ac.kr 